\title{
ON THE LEAST PRIMITIVE ROOT OF A PRIME $p$
}

\section{P. ERDÖS}

Vinogradoff ${ }^{1}$ proved that the least primitive root of a prime $p$ is less than $2^{m} p^{1 / 2} \log p$ for sufficiently large $p ; m$ denotes the number of different prime factors of $p-1$. Later he improved this to $2^{m} p^{1 / 2} \log \log p .{ }^{2} \mathrm{Hua}^{3}$ improved this to $2^{m+1} p^{1 / 2}$. In the present note we are going to prove that the least primitive root is less than $p^{1 / 2}(\log p)^{17}$ for large $p$. This result is better than Hua's if $p-1$ has "many" prime factors, but worse if it has "few" prime factors. We shall use Brun's method. It is very likely that the least primitive root is $o\left(p^{e}\right)$ but I can not even prove that it is less than $c p^{1 / 2}$.

Lemma 1. Let $x<p, k \mid p-1$. Denote by $f_{k}(x)$ the number of $k$ th power residues not exceeding $x$. Then $f_{k}(x)=x / k+O\left(p^{1 / 2} \log p\right)$.

Denote by $\chi_{0}(a), \chi_{1}(a), \cdots, \chi_{k-1}(a)$ the characters for which $\left[\chi_{i}(a)\right]^{k}=1 . \chi_{0}(a)$ is the principal character. Clearly $\sum_{i=0}^{k-1}\left[\chi_{i}(a)\right]=k$ if $a$ is a $k$ th power residue, and is 0 otherwise. Thus

$$
\frac{1}{k} \sum_{i=0}^{k-1} \sum_{a=1}^{x} \chi_{i}(a)^{\circ}=f_{k}(x) \text {. }
$$

On the other hand by a well known result of Pólya ${ }^{4}$

$$
\left|\sum_{a=1}^{x} \chi_{i}(a)\right|<p^{1 / 2} \log p \quad(i \neq 0) .
$$

Thus combining (1) and (2) we obtain

$$
f_{k}(x)=x / k+c\left(p^{1 / 2} \log p\right), \quad|c| \leqq 1,
$$

which proves the lemma.

Denote by $F(x)$ the number of primitive roots not exceeding $x$. We clearly have by the sieve of Eratosthenes $F(x)=\sum_{d \mid p-1} \mu(d) f_{d}(x)$. Hence clearly

$$
\begin{array}{r}
F(x)>\left(x-\sum_{q \mid p-1}^{\prime} f_{q}(x)+\sum_{q_{1}, q_{2} \mid p-1, q_{1} \neq q_{2}}^{\left.\sum_{q_{1}, q_{2}}(x)-\cdots\right)}\right. \\
-\sum_{q \mid p-1, q>(\log p)^{3}} f_{q}(x)=\sum_{1}-\sum_{2},
\end{array}
$$

Received by the editors April 11, 1944, and, in revised form, July 13, 1944.

${ }^{1}$ Landau, Vorlesungen ïber Zahlentheorie, vol. 2, p. 178.

2 C. R. (Doklady) Acad. Sci. URSS (1936) pp. 7-11.

${ }^{3}$ Bull. Amer. Math. Soc. vol. 48 (1942) pp. 726-730.

${ }^{4}$ Landau, ibid. p. 178. 
where the $q$ 's are primes and the dashes in $\sum_{1}$ indicate that the summation is extended over only the $q<(\log p)^{3}$. By (3)

$$
\sum_{2}<\sum_{q \mid p-1, q>(\log p)^{3}} \frac{x}{q}+2 p^{1 / 2}(\log p)^{2}<\frac{4 x}{(\log p)^{2}}
$$

for $x>p^{1 / 2}(\log p)^{4}$, since the number of different prime factors of $p-1$ is less than $2 \log p$.

Now we estimate $\sum_{1}$. Here we use Brun's method. If we replace $f_{k}(x)$ by $x / k$ the error we make is less than $p^{1 / 2} \log p$ by (3). Thus following Brun's ${ }^{5}$ reasoning (p. 23, equation 21) we obtain

$$
\begin{aligned}
\sum_{1} & >x \cdot 0.3 \prod_{q \mid p-1, q<(\log p)^{3}}\left(1-\frac{1}{q}\right)-c_{1}(\log p)^{15} p^{1 / 2} \log p \\
& >c_{2} \frac{x}{\log \log p}-c_{1} p^{1 / 2}(\log p)^{16.6}
\end{aligned}
$$

We do not give the details since the argument follows literally from Brun's original argument. We make only the following remark. Put

$$
A=x-\sum_{q_{1} \mid p-1}^{\prime}\left[\frac{x}{q_{1}}\right]+\sum_{q_{1}, q_{2} \mid p-1, q_{1} \ngtr q_{2}}^{\prime}\left[\frac{x}{q_{1} q_{2}}\right]-\cdots .
$$

Then Brun obtains (p. 23, equation 21)

$$
A>x \cdot 0.3 \prod_{q \mid p-1, q<(\log p)^{3}}\left(1-\frac{1}{q}\right)-c_{1}(\log p)^{15} .
$$

The error term $c_{1}(\log p)^{15}$ comes from replacing in $(\log p)^{15}$ terms $[x / k]$ by $x / k$ (see p. 22). Our error term $c_{1} p^{1 / 2}(\log p)^{16}$ comes from replacing in $(\log p)^{15}$ terms $f_{k}(x)$ by $x / k$. Thus we obtain from (4) and (5)

$$
F(x)>c_{2} x / \log \log p-c_{1} p^{1 / 2}(\log p)^{16}-4 x /(\log p)^{2}>0
$$

for $x>p^{1 / 2}(\log p)^{17}$, and $p$ sufficiently large, which completes the proof.

Purdue University

- Le crible d'Eratosthene et la theoreme de Goldbach, Skrifter utgit av Videnskapsselskapets I. Christiania Matematich Naturvidenskabelig Klasse (1920) No. 3. See in particular p. 23, formula (21), and the preceding pages. The prime $p_{r}$ in that formula is less than $(\log p)^{3}$ here.

${ }^{6}$ We have $\prod_{p<y}(1-1 / p)>c / \log y$. See, for example, Hardy-Wright, Theory of numbers, p. 349. 